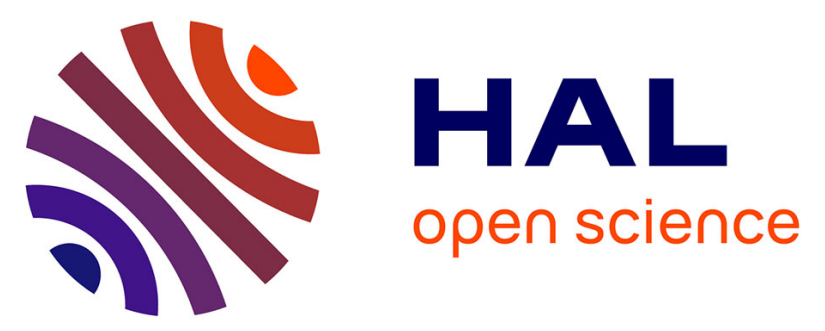

\title{
Sensitivity analysis of HD-sEMG amplitude descriptors relative to grid parameter variations of a cylindrical multilayered muscle model
}

Vincent Carriou, Jeremy Laforet, Sofiane Boudaoud, Mariam Al Harrach

\section{To cite this version:}

Vincent Carriou, Jeremy Laforet, Sofiane Boudaoud, Mariam Al Harrach. Sensitivity analysis of HD-sEMG amplitude descriptors relative to grid parameter variations of a cylindrical multilayered muscle model. Biomedical Physics \& Engineering Express, 2016, 2 (6), pp.064001. 10.1088/20571976/2/6/064001 . hal-01899005

\section{HAL Id: hal-01899005 https://hal.science/hal-01899005}

Submitted on 15 Nov 2018

HAL is a multi-disciplinary open access archive for the deposit and dissemination of scientific research documents, whether they are published or not. The documents may come from teaching and research institutions in France or abroad, or from public or private research centers.
L'archive ouverte pluridisciplinaire HAL, est destinée au dépôt et à la diffusion de documents scientifiques de niveau recherche, publiés ou non, émanant des établissements d'enseignement et de recherche français ou étrangers, des laboratoires publics ou privés. 


\title{
Sensitivity analysis of HD-sEMG amplitude descriptors relative to grid parameter variations of a cylindrical multilayered muscle model
}

\author{
Vincent Carriou ${ }^{1}$, Jeremy Laforet $^{1}$, Sofiane Boudaoud ${ }^{1}$, \\ Mariam Al Harrach ${ }^{1}$ \\ ${ }^{1}$ Sorbonne University, Universite de Technologie de Compiegne, CNRS UMR 7338 \\ Biomechanics and Bioengineering, Centre de Recherche de Royallieu - CS 60203, \\ Compiegne, France
}

E-mail: vincent.carriou@utc.fr

July 2016

\begin{abstract}
The aim of this work is to perform a sensitivity analysis of a high density surface electromyogram (HD-sEMG) amplitude descriptors according to several grid parameters. This study is motivated by the fact that the electrode grid position and layout are crucial to record relevant electromyographic data. For this purpose, an analytical limb model is used, where the upper limb is modeled as a multilayered cylinder with three layers: muscle, fat tissue and skin tissue. Using this model, HD-sEMG signals are computed over the skin as a 2D surface along angular and longitudinal directions. Electrode recording is performed through a surface integration on the 2D surface according to the electrode shape. 16 simulations on 10 anatomies (350 Motor Units) with the same parameters were computed for 3 constant contraction levels: $30 \%, 50 \%$ and $70 \%$ of the Maximal Voluntary Contraction (MVC). Then, a global sensitivity analysis using the Elementary Effect Method is performed to explore the sensitivity of amplitude descriptors (Average Rectified Value, Root Mean Square value and High Order Statistics) relative to varying parameters from the electrode grid (inter-electrode distances, electrodes radius, position and rotation). From those grid definitions, monopolar, bipolar and laplacian signals are also computed to see the electrode arrangement sensitivity. The obtained results exposed a huge impact of the grid rotation on the studied criteria. They also showed that parameters specific to the electrode grid layout (inter-electrode distances) have the less impact. Moreover, they exhibited the laplacian arrangement as the most sensitive electrode arrangement to grid modifications.
\end{abstract}

Keywords: Sensitivity analysis, Amplitude descriptors, HD-sEMG modeling, Elementary effect method 


\section{Introduction}

Mathematical models of surface electromyogram (sEMG) generation consist of a series of equations describing the generation and the propagation of an action potential (AP) along the muscle fibers and within the muscle, respectively [1].

Actually, models allow us the access to electromyographic data corresponding to several physiological contexts and specific motor control strategies that are hardly evaluated in experimental conditions. The investigation of the changes of sEMG signal features according to physiological mechanisms and instrumentation parameters are easier and faster with analytical models rather than numerical models [1, 2].

Recently, a High Density sEMG (HD-sEMG) recording technique has emerged. This technique allows the direct and simultaneous access to a high number of channels (up to 256 channels). This technique significantly increases the spatial representativeness of the recorded data over the studied muscle. It opened the door to multi-channel analysis of muscle activity (as for EEG recordings) and has already demonstrated promising abilities comparing to classical recording techniques [3].

For this purpose, we used a HD-sEMG generation model inspired from a recently published work described in [1]. In this model, the muscle is considered as a cylinder with the possibility of multiple anisotropic layers. The source is described as a progressive generation of the intracellular action potential at the end plate, a propagation along the fiber and its extinction at the ends of the fiber.

Identifying the most influential parameters of the models through some sensitivity analysis is an important task, as it allows one to know (1) where experimental efforts should focus, (2) which parameters are important to identify by inverse methods and (3) on which parameter it is important to perform an uncertainty analysis.

Commonly, the standard deviation of the sEMG signal is used to estimate the magnitude of the muscle activation [4]. Other amplitude estimators, such as Root Mean Square value (RMS) and mean Average Rectified Values (ARV) have been extensively investigated as means of controlling prosthetic devices, ergonomic assessments, biofeedback systems, as well as for a better understanding of healthy and pathological neuromuscular systems [3]. In addition to these classical amplitude parameters, we propose to study features linked to the shape of the sEMG Probability Density Function (PDF), namely, the HOS parameters.

In a previous study, these features have demonstrated, by simulation, promising sensitivity to some sEMG generation parameters [5]. Two High Order Statistics (HOS) will be considered, the Skewness related to the PDF asymmetry and the Kurtosis linked to its flatness.

In this study, we present a global sensitivity analysis of four HD-sEMG amplitude parameters to assess their robustness/sensitiveness, with a parameter ranking, toward the variation of the grid parameters. This task is done using the Elementary Effect Method (EEM) [6] that allows the precise quantification of varying each tested parameters on a specific set of output features [7]. 


\section{Materials and Methods}

In this study we choose to use a screening method as a first approach to a global sensitivity analysis. Screening methods provide a global sensitivity analysis with only a few model runs, hence allowing the analyst to identify parameters on which more complex and costly methods (such as Latin Hypercube sampling) should further focus. We use the toolbox developed in our group as in [8].

In this paper, we will first briefly summarize the model used (previously detailed in [1]) and then describe the screening methodology we apply in section 2.5. Preliminary sensitivity analysis will be presented in section 3 and then discussed.

\subsection{Conductor volume transfer function}

An analytical model describing a muscle as a multilayered cylinder is used in this work [1]. This muscle geometry is reported in Fig. 1, cylindrical coordinates $(\rho ; \theta ; z)$ is used for the calculus and the description of the conductor volume. The origin of the system is located at the center of the cylinder. Moreover, the conductor volume is defined with three layers (muscle, adipose tissue and skin) and sources are located within the most internal layer, along $\mathrm{z}$ axis. All the layers have a finite thickness in the radial direction. Sources are modeled at the microscopic scale and represented fiber intracellular potential generation, propagation and extinction along the fiber. Each layer is homogeneous but may be anisotropic. Thus, muscle tissue has higher conductivity in the longitudinal direction than in the others. The adipous and skin tissues are considered isotropic. We assume that the recording system is placed at the most external boundary (skin-air).

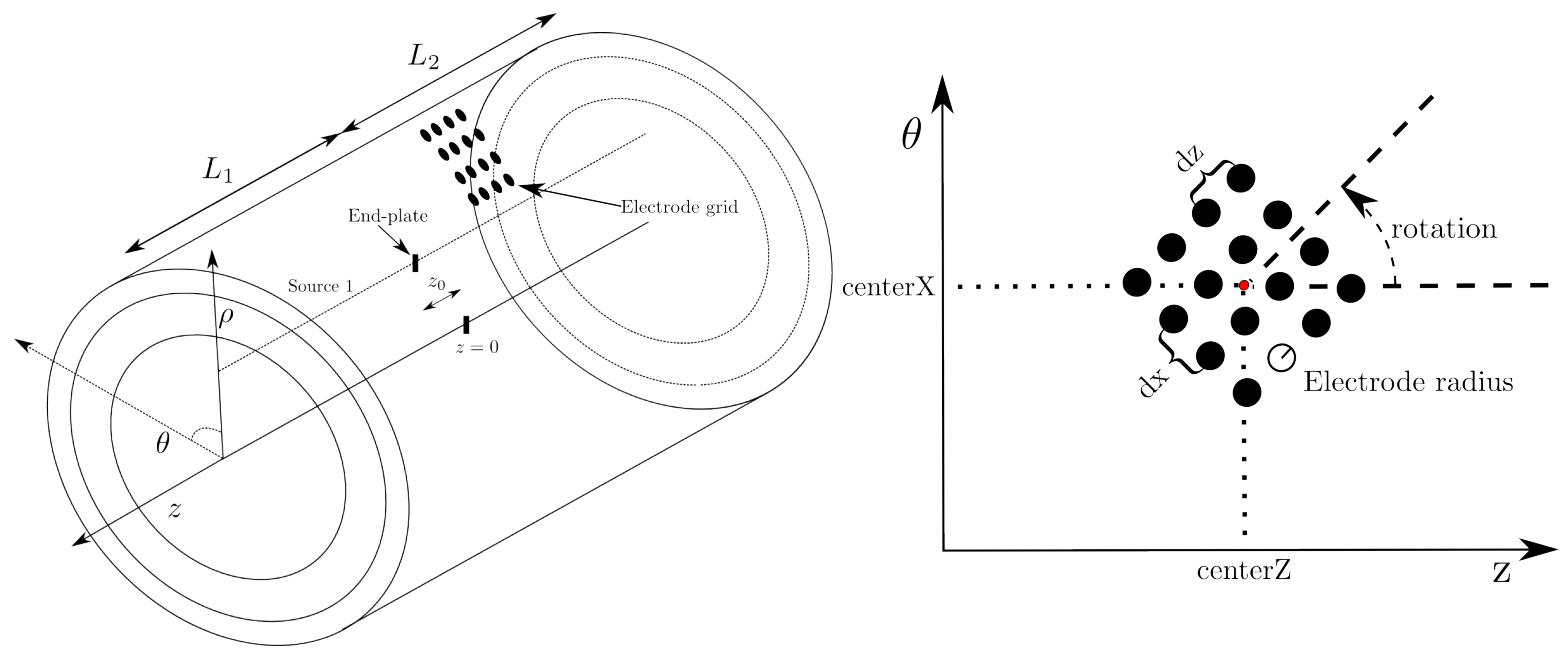

Figure 1: (Left) Cylindrical muscle geometry. $L_{1}$ and $L_{2}$ represent the fiber semilengths according to the neuromuscular junction $\left(z_{0}\right)$. (Right) A $4 \times 4$ electrode grid representation with the studied grid parameters.

Conductor volume transfer function is computed with quasistationnary conditions 
from Poisson equation in cylindrical coordinates:

$$
\nabla . J=-\nabla \cdot(\underline{\underline{\sigma}} \nabla \varphi)=I
$$

where, $J$ is the current density $\left(A \cdot m^{-2}\right) ; \underline{\underline{\sigma}}$ is the conductivity tensor $\left(S . m^{-1}\right), \varphi$ the potential $(\mathrm{V})$ and $I$ the density current of the source $\left(A \cdot \mathrm{m}^{-3}\right)$.

Moreover, boundary conditions between layer and conditions related to the source are imposed. In this model, computation of the sEMG is fully performed in a three dimensional Fourier domain. Details about the resolution of the Poisson equation and computation in Fourier domain are provided in [1].

\subsection{Motor unit and fibers specifications}

In skeletal muscle, four types of motor units (MUs) are displayed within the muscle: Slow (S), Fast Intermediate (FI), Fast Resistant (FR) and Fast Fatigable (FF) MUs [9]. MU territories have circular shapes and are spread randomly with uniform distribution within the muscular area according to its type. Fast MUs are placed closer to the muscle surface than slow MUs in order to mimic suspected MU type repartition in the Biceps Brachii. MU fibers can be mixed with others MU fibers within the muscle. Moreover, possible MU area superposition is also considered in the model. For this purpose, we used a contrained random algorithm presented in [10] which gives more realistic MUs positioning within the muscle. Moreover, boundaries of the muscle are fixed to mimic the Biceps Brachii muscle using MRI technique [11]. Each type of MU has different properties as described in [12. Finally, fibers are defined based on the corresponding MU type. Corresponding muscle fibers are distributed uniformly within each MU territory and fiber conduction velocities are determined as in [12].

\subsection{Motor unit recruitment}

During contraction, MUs are recruited independently of others and based on the size principle. Each MU is activated at a specific threshold depicted in [13].

$$
R T E(i)=e^{a i} ; a=\frac{\ln (R R)}{N}
$$

where, $R T E(i)$ is the recruitment threshold for the $i^{t h} \mathrm{MU}, N$ is the total number of MU and $a$ is a coefficient used to establish a range of threshold values following $N$. It is also tuned by the recruitment range $(R R=80 \%)$ which describes the percentage at which all MUs are recruited. Each MU recruitment is regulated by a motoneuron firing rate which increases linearly with force level, from its minimal firing rate to its peak firing rate.

$$
\begin{aligned}
& F r_{i}(t)=g_{i}\left(E(t)-R T E_{i}\right)+F r_{\text {min }}, \text { if } E(t) \geq R T E_{i} \\
& g_{i}=\frac{F r_{\text {max }_{i}}-F r_{\text {min }_{i}}}{100_{\% M V}-R T E_{i}}
\end{aligned}
$$




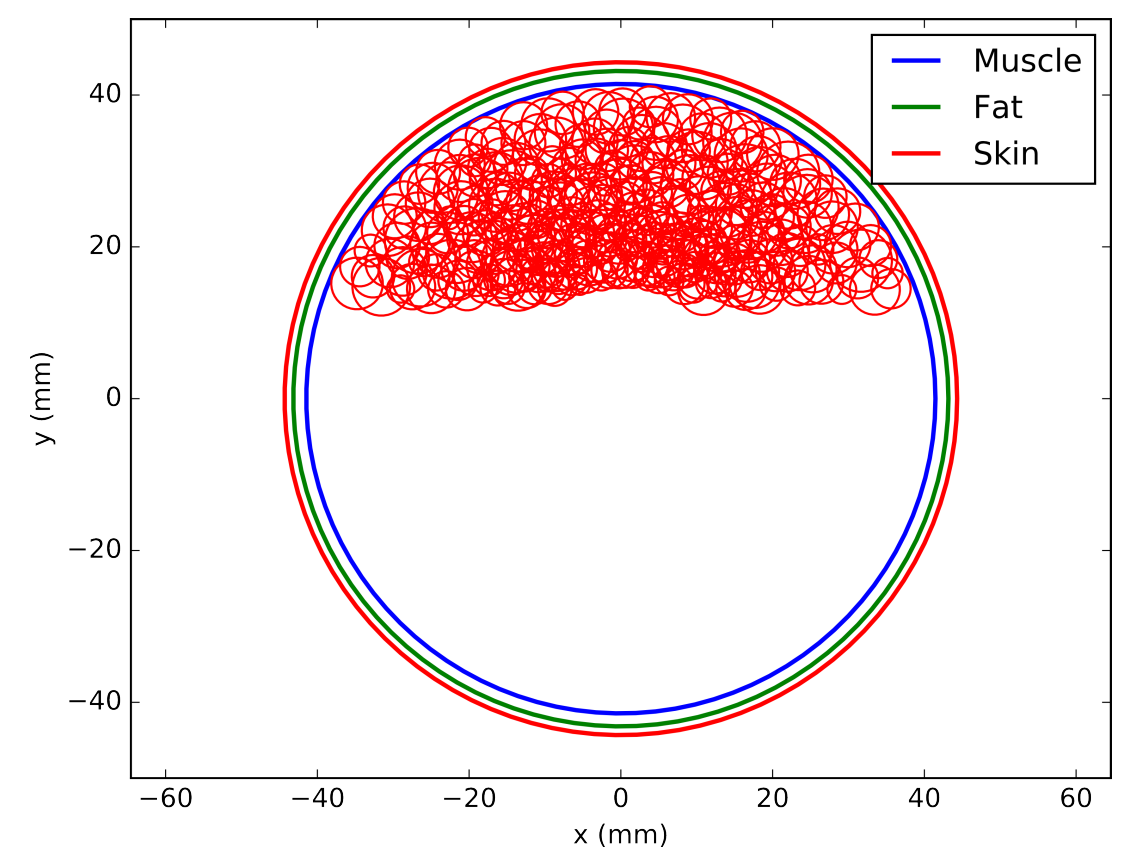

Figure 2: Radial cross-sectional profile of the limb with MUs position within the considered muscle.

$$
F r_{\max _{i}}=F r_{\max _{1}}-P F R D \frac{R T E_{i}}{R T E_{N}}
$$

where, $E(t)$ is the excitatory drive provided by the Central Nervous System (CNS). The firing frequencies $F r_{\text {min }_{i}}$ and $F r_{\max _{1}}$ are fixed to 8 and $35 \mathrm{~Hz}$, respectively. PFRD is the peak firing rate difference and is fixed to $15 \mathrm{~Hz}$ in this study. It accounts for the simulation of the "oignon skin" phenomenon observed in several experimental studies [14].

\subsection{Electrode recording}

Muscle electrical activity is recorded by the electrode at the skin surface with a surfacic integration of the electric activity values over the sampled positions under the electrode. This surface integration is done numericaly and is normalized by the number of sampled positions under the simulated electrode area.

$$
S_{j}\left(\theta_{j}, z_{j}, t_{i}\right)=\iint_{\mathbb{S}_{j}} s E M G\left(\rho_{c}, \theta, z, t_{i}\right) d \theta d z
$$

where $t_{i}$ is an instant point during the simulation, $\left(\theta_{j}, z_{j}\right)$ are the cylindrical coordinates of the center of electrode $j, \mathbb{S}_{j}$ is the electrode area and $\rho_{c}$ is the skin radius. For circular shape electrode, we have:

$$
\mathbb{S}_{j}=\left\{(\theta, z) \mid z^{2}-2 z \cdot z_{j} \cdot \cos \left(\theta-\theta_{j}\right)+z_{j}^{2} \leq r_{j}^{2}\right\}
$$

where, $r_{j}$ is the radius of electrode $j$. 
The principal advantage of the numerical electrode simulation is the total decoupling between the potential map generation and, after, the computing of HDsEMG signals in an infinite number of electrode configurations (number, shape, positions and rotation) without the need of simulating again the potential maps (one for every contraction levels). All the grids in this paper will be composed of $8 \times 8$ electrodes. From this composition we can arrange the monopolar electrodes in the bipolar and laplacian arrangements as we can see on Fig. 3 .

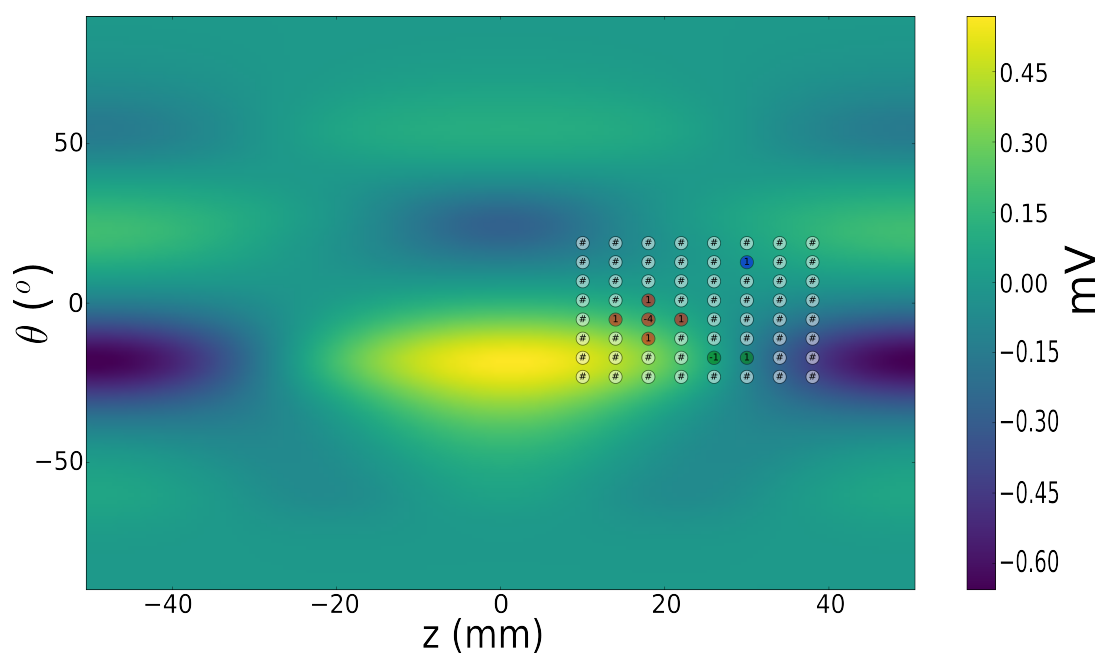

Figure 3: $8 \times 8$ electrode grid placed on the surface of the muscle skin. Blue electrode shows the monopolar arrangement (64 signals). Green electrodes shows the bipolar arrangement with corresponding weight on the electrode (56 signals). Red electrodes sh ows the laplacian arrangement with corresponding weight on the electrode (36 signals).

\subsection{Global sensitivity analysis}

The Elementary Effect Method (EEM), that identifies elementary effects of parameters, is used as a preliminary analysis [6]. This screening method is based on a "one factor at a time" design. It uses local variations (the elementary effects) and averages them over several points in the parameters space in order to obtain a global analysis (see Figure 4). EEM also provides a specific Design Of Experiment (DOE) of the grid parameters from their distributions [6, 15]. In the HD-sEMG model described above, an electrode grid is defined by 6 parameters (see Table 1). Thus, according to [15] 16 electrode grids are defined from the DOE by taking 3 values from the distribution of the parameters. For each simulation, EEM changes the value for one parameter and all the others remain the same. Then, it calculates the outputs changes compared to the other simulation outputs. Finally, EEM determines which parameter is the most sensitive parameter according to the outputs. 
The elementary effects are computed this way:

$$
E E\left(X_{i}^{(j)}\right)=\frac{f\left(X_{1}, \ldots, X_{i}+\Delta, \ldots, X_{n}\right)-f\left(\mathbf{X}^{(j)}\right)}{\Delta}
$$

with $\mathbf{X}^{(j)}=X_{1}, \ldots, X_{i}, \ldots, X_{n}$ is one vector of parameters, $f$ the electrode grid definition and $\Delta$ the parameter variation.

As suggested by [16], we computed the 3 indicators $\mu^{*}, \mu$ and $\sigma$ as follow for a given parameter $i$ :

$$
\begin{aligned}
\mu_{i}^{*} & =\frac{1}{r} \sum_{j=0}^{r}\left|E E\left(X_{i}^{(j)}\right)\right| \\
\mu_{i} & =\frac{1}{r} \sum_{j=0}^{r} E E\left(X_{i}^{(j)}\right) \\
\sigma_{i} & =\sqrt{\frac{1}{r-1} \sum_{j=0}^{r}\left(E E\left(X_{i}^{(j)}\right)-\mu_{i}\right)^{2}}
\end{aligned}
$$

with $r$ the number of electrode grid on which the elementary effect is computed. Indicators $\mu^{*}$ and $\mu$ represent the direct interaction of the parameter on the outputs of the model and $\sigma$ defines the possible indirect interaction of the parameter on the outputs through interactions with the other parameters.

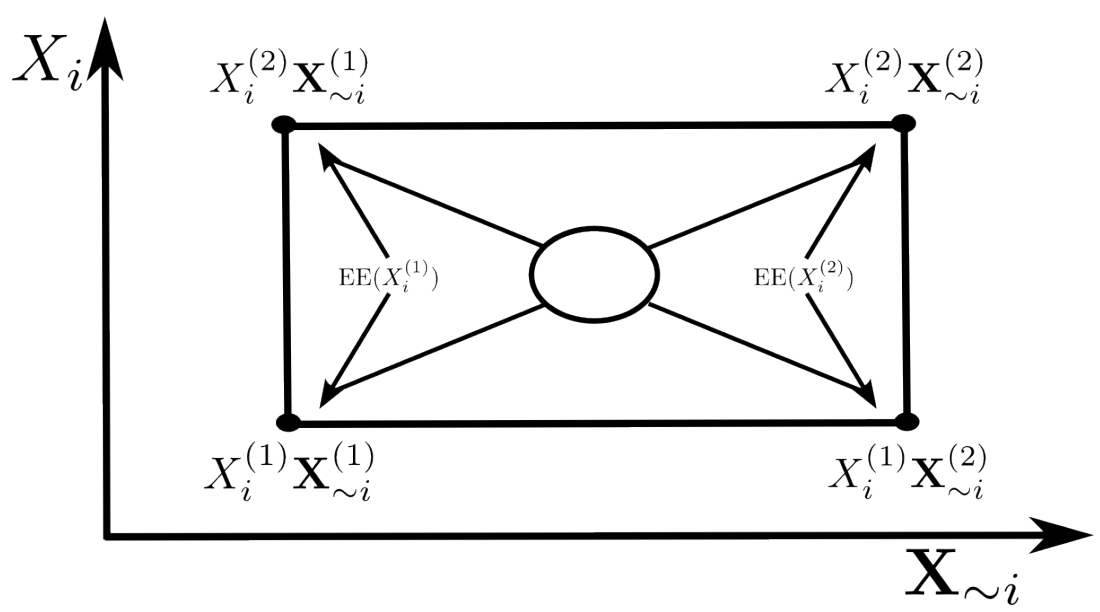

Figure 4: Elementary Effect (EE) computation diagram. $X_{i}^{1}$ and $X_{i}^{2}$ are two different values of the parameter $X_{i} . \mathbf{X}_{\sim i}^{(1)}$ and $\mathbf{X}_{\sim i}^{(2)}$ are two different parameter sets excluding parameter $X_{i} . E E\left(X_{i}^{(j)}\right)$ is the Elementary Effect of the tested parameter $X_{i}^{(j)}$ value with the $j^{\text {th }}$ value of the parameter set. $X_{i}^{(1)} \mathbf{X}_{\sim i}^{(1)}$ corresponds to one set of parameter, $X_{i}^{(2)} \mathbf{X}_{\sim i}^{(1)}$ is equivalent to the same set of parameter with a different value of the parameter $X_{i}$.

Once the features are computed for all the sets of parameters, they are ranked by their sensitivity index (SI) computed from $\sqrt{\mu^{* 2}+\sigma^{2}}$ (see Figure 5 ) for each features. 


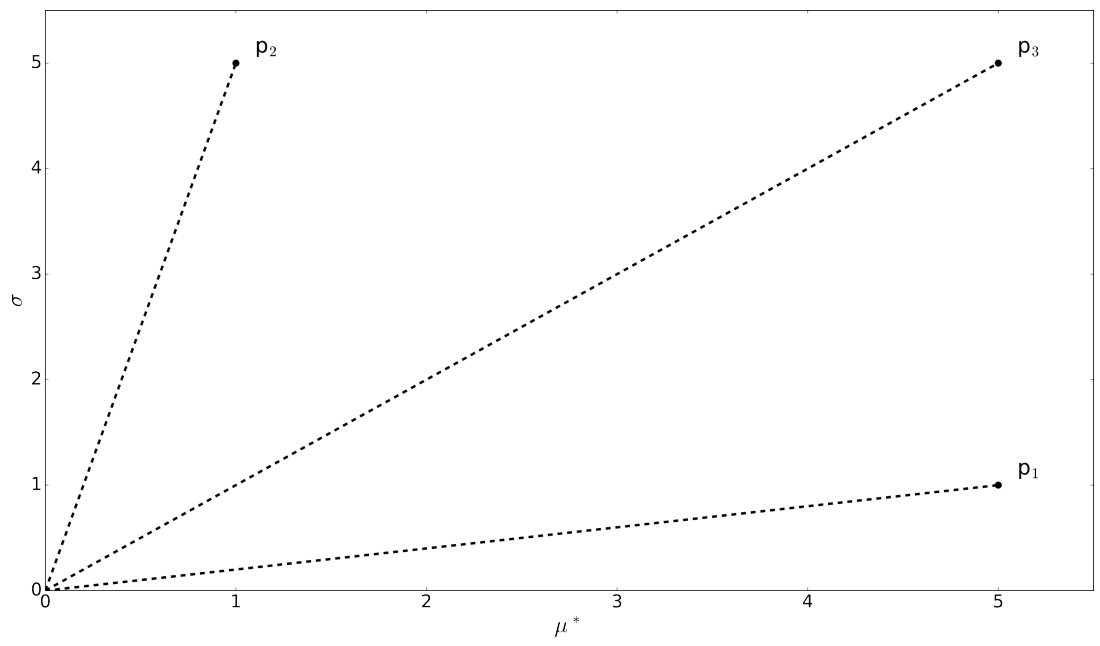

Figure 5: Example of sensitivity index for 3 parameters. The parameter $\mathrm{p}_{1}$ has a higher direct impact on the outputs of the simulation than the parameter $\mathrm{p}_{2}\left(\mu_{1}^{*}>\mu_{2}^{*}\right)$. Conversely, the parameter $\mathrm{p}_{2}$ has an higher impact through the other parameters of the simulation on the outputs than the parameter $\mathrm{p}_{1}\left(\sigma_{2}>\sigma_{1}\right)$. However, the most sensitive parameter here is the parameter $\mathrm{p}_{3}$.

Each SI can be represented on a figure and corresponds to the distance between the origin and the corresponding parameter point placed at its coordinates $\left(\mu^{*} ; \sigma\right)$.

The Figure 5 exhibits a simple example of SI for three parameters. The parameters $\mathrm{p}_{1}, \mathrm{p}_{2}$ and $\mathrm{p}_{3}$ have $(5 ; 1),(1 ; 5)$ and $(5 ; 5)$ for $\left(\mu^{*} ; \sigma\right)$ values, respectively. By computing the SI for each parameters, we see that the most sensitive parameter is $\mathrm{p}_{3}$ with a SI of 7.07. Moreover, parameters $\mathrm{p}_{1}$ and $\mathrm{p}_{2}$ have the same SI of 5.10 but have different values of $\left(\mu^{*} ; \sigma\right)$ and thus different effects on the features. On the Figure 5 it is not clear that the parameters $\mathrm{p}_{1}$ and $\mathrm{p}_{2}$ have the same SI. Thus, this kind of representation can be hard to interpret due to the scales of the axes. If the axes scales are not the same, two parameters can have the same SI but distance from origin can appear different on this type of figure (as on the figure 5 between parameters $\mathrm{p}_{1}$ and $\mathrm{p}_{2}$ ). Finally, this representation loses information from the sensitivity analysis such as the monotonous effect of a parameter if $\mu^{*}=|\mu|$.

As indicated above, the parameters of the electrode grid have to be defined using specific distribution for each parameter. These parameters with their variation ranges for the sensitivity analysis are summarized in table 1 . 


\begin{tabular}{|cl|c|c|}
\hline Name & Description & Variables distribution & Unit \\
\hline $\mathrm{dx}$ & Angular inter-electrode distance & $\mathrm{U}(4.0 ; 6.0)$ & $\mathrm{mm}$ \\
$\mathrm{dz}$ & Longitudinal inter-electrode distance & $\mathrm{U}(4.0 ; 6.0)$ & $\mathrm{mm}$ \\
$\mathrm{r}$ & Electrode radius & $\mathrm{U}(0.5 ; 2.0)$ & $\mathrm{mm}$ \\
centerX & Angular electrode grid center & $\mathrm{T}(-10 ; 10 ; 0)$ & $o$ \\
centerZ & Longitudinal electrode grid center & $\mathrm{T}(23.25 ; 27.25 ; 25.25)$ & $\mathrm{mm}$ \\
rotation & Electrode grid rotation & $\mathrm{T}(-15 ; 15 ; 0)$ & $o$ \\
\hline
\end{tabular}

Table 1: Parameters used for sensitivity analysis and their variation range.

Where, $\mathrm{U}(\mathrm{a} ; \mathrm{b})$ represents the uniform distribution on $[a ; b] . \mathrm{T}(\mathrm{a} ; \mathrm{b} ; \mathrm{c})$ represents the triangular distribution on $[a ; b]$ with a peak at $c \in[a ; b]$.

As presented in the introduction, the purpose of this study is to assess which parameter of the grid has the higher impact on the signal features and thus which study can be compared with others.

We assume no prior knowledge on the possible variations of the layout parameters of the grid (inter-electrode distances and electrode radius), hence we use uniform distributions. Nevertheless, we assume a realistic range of values for these parameters. We used a triangular distribution for the position parameters of the grid (grid center position and rotation). This kind of distribution assess the highest probability for the value $c$ with linear decreasing probability to the values $a$ and $b$. We used this distribution to model the possible deviation of the grid position according to a desired position. Range of values chosen for these parameters is in respect to the protocol for the HDsEMG recording on the Biceps Brachii [17].

\subsection{Signal features}

We computed the elementary effects not on the signals themselves but on features extracted from these signals. Root Mean Square (RMS), Average Rectified Value (ARV) and High Order Statistics (HOS) are used as output features. Outputs of the model used for the analysis are statistical criteria on the signal recorded by the electrodes.

- RMS amplitude of signal $Y: R M S(Y)=\sqrt{\frac{1}{n} \sum_{j=0}^{n} Y(j)^{2}}$;

- ARV amplitude of signal $Y: A R V(Y)=\frac{1}{n} \sum_{j=0}^{n}|Y(j)|$;

- Kurtosis of signal $Y: \operatorname{Kur}(Y)=\frac{E\left(Y-\mu_{Y}\right)^{4}}{\sigma_{Y}^{4}}-3$;

- Skewness of signal $Y: \operatorname{Skew}(Y)=\frac{E\left(Y-\mu_{Y}\right)^{3}}{\sigma_{Y}^{3}}$.

With $n$ the number of sampling points in the signal, $E($.$) the expectation operator$ and $\mu_{Y}, \sigma_{Y}$ the expected values and standard deviation of the signal $Y$, respectively. 
Those features have been used in previous studies [18] and are classical amplitude statistic descriptors on the sEMG signals.

\section{Results}

All the simulations were performed on a dedicated workstation $2 \times 8$ cores Intel Xeon 2.40Ghz with hyperthreading, 128Gb Ram, Ubuntu 14.04 64bits). The 16 grid definitions needed to compute the elementary effects of all parameters were obtained in a few seconds. Signals recorded by the 16 electrode grids are performed on the same simulated 2D surfacic potential maps. Statistics are computed for monopolar, bipolar and laplacian electrode arrangements. Then, statistics are computed for the 64 monopolar (56 bipolar and 36 laplacian) signals of the grid and the mean value over the electrodes is used. Ten anatomies with the same properties presented in table 2 were computed to have robust results. The ANOVA test is computed for the 10 anatomies for each features and proves that there is no significant influence of the anatomies on the mean value of the studied features. Thus, the sensitivity measures are computed for the features for each anatomy and then the mean value of the features is computed from the 10 anatomies. Since, the sensitivity measures give almost the same results in these two cases, we will only present the results for the mean value of the features on the 10 anatomies.

\begin{tabular}{|cl|c|}
\hline Name & Description & Parameter Value (Unit) \\
\hline nMUs & Number of MUs & 352 \\
nFibs & Number of fibers & 60862 \\
$\rho_{m}$ & Muscle radius & $42(\mathrm{~mm})$ \\
$\sigma^{m}$ & Radial and angular muscle conductivity & $0.1\left(\mathrm{~S}^{-1}\right)$ \\
$\sigma_{z}^{m}$ & Longitudinal muscle conductivity & $0.5\left(\mathrm{~S}^{-1}\right)$ \\
$\rho_{a p}$ & Adipose tissue thickness & $2(\mathrm{~mm})$ \\
$\sigma^{a p}$ & Adipose tissue conductivity & $0.05\left(\mathrm{~S} . \mathrm{m}^{-1}\right)$ \\
$\rho_{s}$ & Skin thickness & $1(\mathrm{~mm})$ \\
$\sigma^{s}$ & Skin conductivity & $1\left({\left.\mathrm{~S} . \mathrm{m}^{-1}\right)}\right.$ \\
\hline
\end{tabular}

Table 2: Parameters used for the generation of the ten anatomies.

\subsection{Monopolar sensitivity ranking}

We present in tables 3 and 4 all the parameter sensitivities computed for each signal features by the elementary effect method previously described. Signal features are computed from the mean of the corresponding features of the ten anatomies. For nearly all parameters $\mu^{*}$ equals to $|\mu|$, regardless of the feature. This means that the EE computed at different points of the parameter space have the same sign, indicating monotonous 
effects.

The Fig.6 presents the ranked parameters according to their sensitivity in descending order for each features for the monopolar arrangement. All the features agree that the three most sensitive parameters are the rotation, the lateral center of the grid and the electrode radius. Moreover, the two least sensitive parameters are the lateral and the longitudinal inter-electrode distance variation. Only the Skewness at 30 $\% \mathrm{MVC}$ shows different ranking for the parameter. This can be linked to the less number of recruited MUs at this contraction level which seems to highly interfer with the PDF assymmetry.

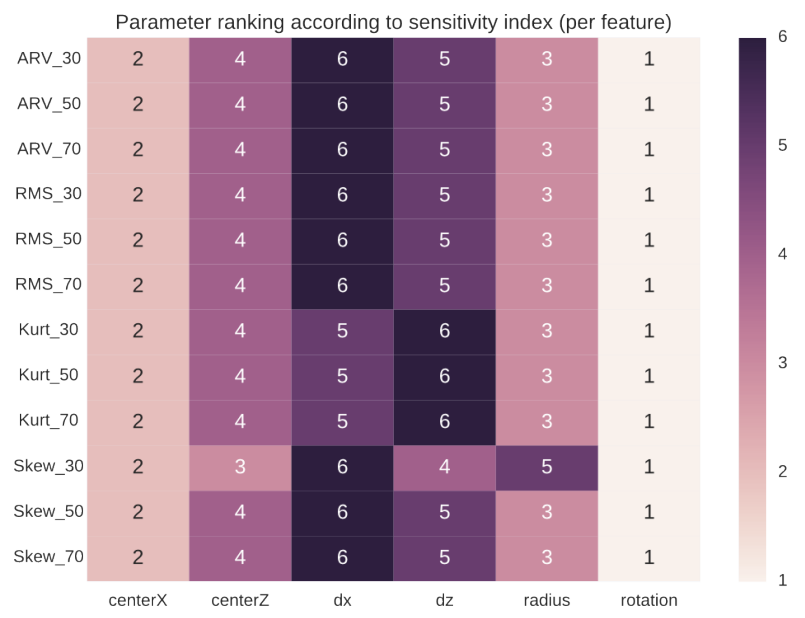

Figure 6: Parameters ranking according to the features for monopolar arrangement on the grid.

Parameters are not considered sensitive when their $\mu^{*}$ is a 100 times smaller than the maximal $\mu^{*}$ for the given feature [6, 15]. According to the values presented in tables 3 and 4, we don't observe significant difference between the $\mu^{*}$ values which means that all the parameters are sensitive for the given features. For monopolar arrangement, the rotation, the electrode radius and the lateral inter-electrode distance $(\mathrm{dx})$ show a difference between $\mu^{*}$ and $|\mu|$ for the Skewness at $30 \%$ MVC only (see values highlight in red). This means that those parameters have non-monotonous effects on the feature. Moreover, no parameter has a high $\sigma$ value. It means that there is no indirect effects on the features through interactions with other parameters.

To conclude on the monopolar arrangement, ARV and RMS features show very similar sensitivity ranking and measures for monopolar arrangement, showing that these two features are equivalent for our study. Moreover, sensitivity measure tables by anatomies show different ranking for the rotation and the lateral center of the grid (centerX) for the Skewness at 30\%MVC feature. Thus, they present differences in the parameters ranking, the longitudinal center of the grid (centerZ) has a higher impact than the lateral center (centerX) for one anatomy for the Skewness at 30\%MVC. 


\begin{tabular}{|c|c|r|r|r|}
\hline rk & Param. & $\mu^{*}$ & $\mu$ & $\sigma$ \\
\hline 1 & rotation & 0.2014 & 0.2014 & $7.66 .10^{-5}$ \\
2 & centerX & 0.1870 & 0.1870 & $2.23 .10^{-5}$ \\
3 & radius & 0.1097 & -0.1097 & $4.06 .10^{-6}$ \\
4 & centerZ & 0.0615 & 0.0615 & $1.28 .10^{-6}$ \\
5 & $\mathrm{dz}$ & 0.0344 & -0.0344 & $1.42 .10^{-7}$ \\
6 & $\mathrm{dx}$ & 0.0247 & -0.0247 & $8.02 .10^{-7}$ \\
\hline
\end{tabular}

(a) ARV at $30 \%$ MVC.

\begin{tabular}{|c|c|r|r|r|}
\hline rk & Param. & $\mu^{*}$ & $\mu$ & $\sigma$ \\
\hline 1 & rotation & 0.5334 & 0.5334 & $6.12 .10^{-5}$ \\
2 & centerX & 0.5016 & 0.5016 & $1.87 .10^{-5}$ \\
3 & radius & 0.2842 & -0.2842 & $3.36 .10^{-6}$ \\
4 & centerZ & 0.1618 & 0.1618 & $2.92 .10^{-6}$ \\
5 & $\mathrm{dz}$ & 0.0780 & -0.0780 & $1.07 .10^{-6}$ \\
6 & $\mathrm{dx}$ & 0.0649 & -0.0649 & $5.56 .10^{-7}$ \\
\hline
\end{tabular}

(c) ARV at $70 \% \mathrm{MVC}$.

\begin{tabular}{|c|c|r|r|r|}
\hline rk & Param. & $\mu^{*}$ & $\mu$ & $\sigma$ \\
\hline 1 & rotation & 0.2624 & 0.2624 & $6.40 .10^{-5}$ \\
2 & centerX & 0.2441 & 0.2441 & $1.61 .10^{-5}$ \\
3 & radius & 0.1391 & -0.1391 & $4.52 .10^{-6}$ \\
4 & centerZ & 0.0814 & 0.0814 & $5.17 .10^{-6}$ \\
5 & $\mathrm{dz}$ & 0.0443 & -0.0443 & $1.71 .10^{-6}$ \\
6 & $\mathrm{dx}$ & 0.0318 & -0.0318 & $3.52 .10^{-8}$ \\
\hline
\end{tabular}

(e) RMS at $50 \%$ MVC.

\begin{tabular}{|c|c|r|r|r|}
\hline rk & Param. & $\mu^{*}$ & $\mu$ & $\sigma$ \\
\hline 1 & rotation & 0.3877 & 0.3877 & $6.80 .10^{-5}$ \\
2 & centerX & 0.3630 & 0.3630 & $1.99 .10^{-5}$ \\
3 & radius & 0.2078 & -0.2078 & $3.95 .10^{-6}$ \\
4 & centerZ & 0.1181 & 0.1181 & $3.02 .10^{-6}$ \\
5 & $\mathrm{dz}$ & 0.0584 & -0.0584 & $6.16 .10^{-7}$ \\
6 & $\mathrm{dx}$ & 0.0470 & -0.0470 & $1.67 .10^{-8}$ \\
\hline
\end{tabular}

(b) ARV at $50 \% \mathrm{MVC}$.

\begin{tabular}{|c|c|r|r|r|}
\hline rk & Param. & $\mu^{*}$ & $\mu$ & $\sigma$ \\
\hline 1 & rotation & 0.0660 & 0.0660 & $3.40 .10^{-5}$ \\
2 & centerX & 0.0600 & 0.0600 & $9.06 .10^{-6}$ \\
3 & radius & 0.0366 & -0.0366 & $1.99 .10^{-6}$ \\
4 & centerZ & 0.0206 & 0.0206 & $6.13 .10^{-7}$ \\
5 & $\mathrm{dz}$ & 0.0146 & -0.0146 & $5.47 .10^{-8}$ \\
6 & $\mathrm{dx}$ & 0.0081 & -0.0081 & $3.93 .10^{-7}$ \\
\hline
\end{tabular}

(d) RMS at $30 \%$ MVC.

\begin{tabular}{|c|c|r|r|r|}
\hline rk & Param. & $\mu^{*}$ & $\mu$ & $\sigma$ \\
\hline 1 & rotation & 0.4459 & 0.4459 & $1.37 .10^{-4}$ \\
2 & centerX & 0.4169 & 0.4169 & $3.78 .10^{-5}$ \\
3 & radius & 0.2359 & -0.2359 & $8.83 .10^{-6}$ \\
4 & centerZ & 0.1377 & 0.1377 & $9.34 .10^{-6}$ \\
5 & $\mathrm{dz}$ & 0.0749 & -0.0749 & $3.87 .10^{-6}$ \\
6 & $\mathrm{dx}$ & 0.0538 & -0.0538 & $8.55 .10^{-7}$ \\
\hline
\end{tabular}

(f) RMS at $70 \% \mathrm{MVC}$.

Table 3: Detailed monopolar ARV and RMS features sensitivity for all parameters on the mean features of the ten anatomies. Highlighted values in red correspond to values indicating a non monotonous effect of the parameter. 


\begin{tabular}{|c|c|r|r|r|}
\hline rk & Param. & $\mu^{*}$ & $\mu$ & $\sigma$ \\
\hline 1 & rotation & 1.4678 & 1.4678 & $2.02 .10^{-4}$ \\
2 & centerX & 1.3583 & 1.3583 & $2.80 .10^{-4}$ \\
3 & radius & 0.7635 & -0.7635 & $2.39 .10^{-4}$ \\
4 & centerZ & 0.4205 & 0.4205 & $8.81 .10^{-7}$ \\
5 & $\mathrm{dx}$ & 0.1772 & -0.1772 & $6.80 .10^{-5}$ \\
6 & $\mathrm{dz}$ & 0.1591 & -0.1591 & $4.15 .10^{-5}$ \\
\hline
\end{tabular}

(a) Kurtosis at $30 \%$ MVC.

\begin{tabular}{|c|c|r|r|r|}
\hline rk & Param. & $\mu^{*}$ & $\mu$ & $\sigma$ \\
\hline 1 & rotation & 1.4192 & 1.4192 & $2.12 .10^{-3}$ \\
2 & centerX & 1.3424 & 1.3424 & $1.55 .10^{-3}$ \\
3 & radius & 0.6906 & -0.6906 & $1.00 .10^{-6}$ \\
4 & centerZ & 0.4080 & 0.4080 & $1.00 .10^{-5}$ \\
5 & $\mathrm{dx}$ & 0.1697 & -0.1697 & $9.10 .10^{-5}$ \\
6 & $\mathrm{dz}$ & 0.1299 & -0.1299 & $3.10 .10^{-5}$ \\
\hline
\end{tabular}

(c) Kurtosis at $70 \%$ MVC.

\begin{tabular}{|c|c|r|r|r|}
\hline rk & Param. & $\mu^{*}$ & $\mu$ & $\sigma$ \\
\hline 1 & rotation & 0.7000 & -0.7000 & $1.90 .10^{-5}$ \\
2 & centerX & 0.6469 & -0.6469 & $8.00 .10^{-5}$ \\
3 & radius & 0.3439 & 0.3439 & $4.80 .10^{-5}$ \\
4 & centerZ & 0.1972 & -0.1972 & $9.50 .10^{-5}$ \\
5 & $\mathrm{dz}$ & 0.0889 & 0.0889 & $8.40 .10^{-5}$ \\
6 & $\mathrm{dx}$ & 0.0784 & 0.0784 & $2.60 .10^{-5}$ \\
\hline
\end{tabular}

(e) Skewness at $50 \% \mathrm{MVC}$.

\begin{tabular}{|c|c|r|r|r|}
\hline rk & Param. & $\mu^{*}$ & $\mu$ & $\sigma$ \\
\hline 1 & rotation & 3.7488 & 3.7488 & 0.0342 \\
2 & centerX & 3.5567 & 3.5567 & 0.0249 \\
3 & radius & 1.8030 & -1.8030 & $1.98 .10^{-4}$ \\
4 & centerZ & 1.0699 & 1.0699 & $1.01 .10^{-4}$ \\
5 & $\mathrm{dx}$ & 0.4271 & -0.4271 & $7.40 .10^{-5}$ \\
6 & $\mathrm{dz}$ & 0.2878 & -0.2878 & $3.21 .10^{-4}$ \\
\hline
\end{tabular}

(b) Kurtosis at $50 \% \mathrm{MVC}$.

\begin{tabular}{|c|c|r|r|r|}
\hline rk & Param. & $\mu^{*}$ & $\mu$ & $\sigma$ \\
\hline 1 & rotation & 0.0560 & 0.0195 & $3.14 .10^{-3}$ \\
2 & centerX & 0.0468 & 0.0468 & $5.62 .10^{-4}$ \\
3 & centerZ & 0.0350 & 0.0350 & $1.44 .10^{-4}$ \\
4 & $\mathrm{dz}$ & 0.0299 & 0.0299 & $1.00 .10^{-6}$ \\
5 & radius & 0.0162 & 0.0012 & $2.61 .10^{-4}$ \\
6 & $\mathrm{dx}$ & 0.0090 & -0.0062 & $8.20 .10^{-5}$ \\
\hline
\end{tabular}

(d) Skewness at $30 \%$ MVC.

\begin{tabular}{|c|c|r|r|r|}
\hline rk & Param. & $\mu^{*}$ & $\mu$ & $\sigma$ \\
\hline 1 & rotation & 0.4176 & -0.4176 & $1.73 .10^{-5}$ \\
2 & centerX & 0.3895 & -0.3895 & $5.86 .10^{-7}$ \\
3 & radius & 0.2040 & 0.2040 & $1.01 .10^{-5}$ \\
4 & centerZ & 0.1184 & -0.1184 & $2.09 .10^{-5}$ \\
5 & $\mathrm{dz}$ & 0.0562 & 0.0562 & $3.52 .10^{-5}$ \\
6 & $\mathrm{dx}$ & 0.0491 & 0.0491 & $2.43 .10^{-6}$ \\
\hline
\end{tabular}

(f) Skewness at $70 \% \mathrm{MVC}$.

Table 4: Detailed monopolar kurtosis and skewness features sensitivity for all parameters on the mean features of the ten anatomies. Highlighted values in red correspond to values indicating a non monotonous effect of the parameter. 


\subsection{Bipolar sensitivity ranking}

On Fig. 7 is presented the ranking of the most sensitive parameters acoording to the features. As in the monopolar ranking (see Fig. 6), the rotation, the lateral center of the grid and the electrode radius are the three most sensitive parameters. Also, the inter-electrode distances are the two less sensitive according to the results. However, there are some differences about the skewness feature at 50 and $70 \% \mathrm{MVC}$. The longitudinal center and the longitudinal inter-electrode distance is more sensitive with the bipolar arrangement than in the monopolar and laplacian. It can be explained by the longitudinal bipolar arrangement of the electrode and also because it is supposed to be aligned with muscle fibers.

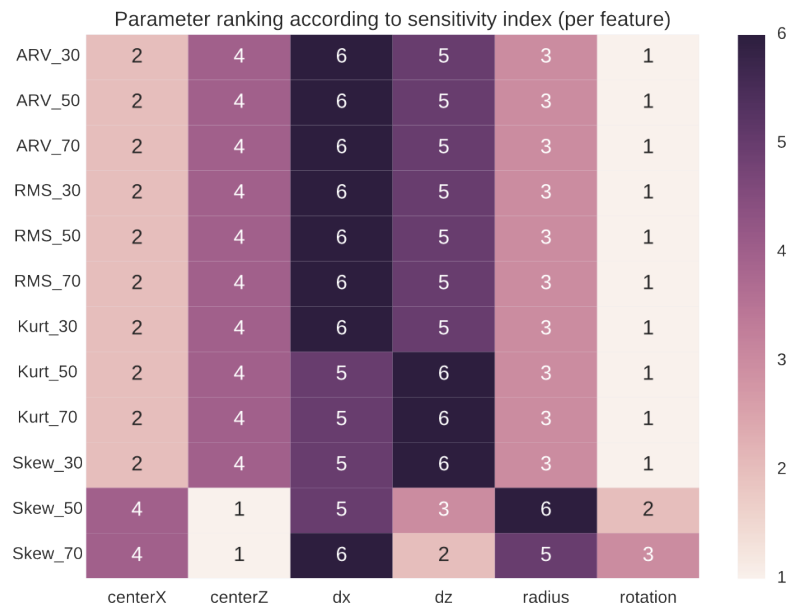

Figure 7: Parameters ranking according to the features for bipolar arrangement on the grid.

According to the tables 5 and 6. we can assess that all the grid parameters are sensitive for the features. As in the monopolar results, no parameter has a high value of $\sigma$. With a deeper insight into the results by anatomies, we observe several differences in the ranking of the parameters for the skewness features at 50 and $70 \% \mathrm{MVC}$. In fact, there is only a factor of around 4 and 6 between the most sensitive parameter and the less at 50 and $70 \% \mathrm{MVC}$, respectively. Thus, the skewness features at 50 and $70 \% \mathrm{MVC}$ seem to be sensitive to the anatomy with the longitudinal bipolar arrangement. 


\begin{tabular}{|c|c|r|r|r|}
\hline rk & Param. & $\mu^{*}$ & $\mu$ & $\sigma$ \\
\hline 1 & rotation & 0.1417 & 0.1417 & $4.28 .10^{-5}$ \\
2 & centerX & 0.1311 & 0.1311 & $1.07 .10^{-5}$ \\
3 & radius & 0.0770 & -0.0770 & $2.53 .10^{-6}$ \\
4 & centerZ & 0.0438 & 0.0438 & $7.20 .10^{-7}$ \\
5 & $\mathrm{dz}$ & 0.0244 & -0.0244 & $1.00 .10^{-7}$ \\
6 & $\mathrm{dx}$ & 0.0175 & -0.0175 & $1.18 .10^{-6}$ \\
\hline
\end{tabular}

(a) ARV at $30 \%$ MVC.

\begin{tabular}{|c|c|r|r|r|}
\hline rk & Param. & $\mu^{*}$ & $\mu$ & $\sigma$ \\
\hline 1 & rotation & 0.4055 & 0.4055 & $1.24 .10^{-4}$ \\
2 & centerX & 0.3763 & 0.3763 & $1.30 .10^{-5}$ \\
3 & radius & 0.2149 & -0.2149 & $1.30 .10^{-5}$ \\
4 & centerZ & 0.1294 & 0.1294 & $7.00 .10^{-6}$ \\
5 & $\mathrm{dz}$ & 0.0696 & -0.0696 & $1.00 .10^{-6}$ \\
6 & $\mathrm{dx}$ & 0.0491 & -0.0491 & $8.00 .10^{-6}$ \\
\hline
\end{tabular}

(c) ARV at $70 \% \mathrm{MVC}$.

\begin{tabular}{|c|c|r|r|r|}
\hline rk & Param. & $\mu^{*}$ & $\mu$ & $\sigma$ \\
\hline 1 & rotation & 0.1615 & 0.1615 & $1.15 .10^{-4}$ \\
2 & centerX & 0.1453 & 0.1453 & $1.16 .10^{-5}$ \\
3 & radius & 0.0843 & -0.0843 & $1.23 .10^{-5}$ \\
4 & centerZ & 0.0546 & 0.0546 & $7.64 .10^{-6}$ \\
5 & $\mathrm{dz}$ & 0.0378 & -0.0378 & $7.51 .10^{-7}$ \\
6 & $\mathrm{dx}$ & 0.0192 & -0.0192 & $2.06 .10^{-6}$ \\
\hline
\end{tabular}

(e) RMS at $50 \%$ MVC.

\begin{tabular}{|c|c|r|r|r|}
\hline rk & Param. & $\mu^{*}$ & $\mu$ & $\sigma$ \\
\hline 1 & rotation & 0.2889 & 0.2889 & $8.83 .10^{-5}$ \\
2 & centerX & 0.2666 & 0.2666 & $8.68 .10^{-6}$ \\
3 & radius & 0.1538 & -0.1538 & $1.01 .10^{-5}$ \\
4 & centerZ & 0.0916 & 0.0916 & $5.14 .10^{-6}$ \\
5 & $\mathrm{dz}$ & 0.0496 & -0.0496 & $6.54 .10^{-7}$ \\
6 & $\mathrm{dx}$ & 0.0351 & -0.0351 & $3.02 .10^{-6}$ \\
\hline
\end{tabular}

(b) ARV at $50 \% \mathrm{MVC}$.

\begin{tabular}{|c|c|r|r|r|}
\hline rk & Param. & $\mu^{*}$ & $\mu$ & $\sigma$ \\
\hline 1 & rotation & 0.0314 & 0.0314 & $9.66 .10^{-6}$ \\
2 & centerX & 0.0282 & 0.0282 & $2.10 .10^{-6}$ \\
3 & radius & 0.0174 & -0.0174 & $6.58 .10^{-7}$ \\
4 & centerZ & 0.0100 & 0.0100 & $1.80 .10^{-7}$ \\
5 & $\mathrm{dz}$ & 0.0073 & -0.0073 & $1.14 .10^{-8}$ \\
6 & $\mathrm{dx}$ & 0.0039 & -0.0039 & $2.24 .10^{-7}$ \\
\hline
\end{tabular}

(d) RMS at $30 \% \mathrm{MVC}$.

\begin{tabular}{|c|c|r|r|r|}
\hline rk & Param. & $\mu^{*}$ & $\mu$ & $\sigma$ \\
\hline 1 & rotation & 0.2725 & 0.2725 & $2.97 .10^{-4}$ \\
2 & centerX & 0.2473 & 0.2473 & $3.90 .10^{-5}$ \\
3 & radius & 0.1425 & -0.1425 & $2.80 .10^{-5}$ \\
4 & centerZ & 0.0922 & 0.0922 & $1.60 .10^{-5}$ \\
5 & $\mathrm{dz}$ & 0.0638 & -0.0638 & $2.00 .10^{-6}$ \\
6 & $\mathrm{dx}$ & 0.0320 & -0.0320 & $9.00 .10^{-6}$ \\
\hline
\end{tabular}

(f) RMS at $70 \% \mathrm{MVC}$.

Table 5: Detailed bipolar ARV and RMS features sensitivity for all parameters on the mean features of the ten anatomies. Highlighted values in red correspond to values indicating a non monotonous effect of the parameter. 


\begin{tabular}{|c|c|r|r|r|}
\hline rk & Param. & $\mu^{*}$ & $\mu$ & $\sigma$ \\
\hline 1 & rotation & 1.4955 & 1.4955 & $5.33 .10^{-3}$ \\
2 & centerX & 1.3621 & 1.3621 & $2.62 .10^{-4}$ \\
3 & radius & 0.7947 & -0.7947 & $8.00 .10^{-4}$ \\
4 & centerZ & 0.4419 & 0.4419 & $5.00 .10^{-6}$ \\
5 & $\mathrm{dz}$ & 0.2453 & -0.2453 & $2.10 .10^{-5}$ \\
6 & $\mathrm{dx}$ & 0.1769 & -0.1769 & $2.97 .10^{-4}$ \\
\hline
\end{tabular}

(a) Kurtosis at $30 \%$ MVC.

\begin{tabular}{|c|c|r|r|r|}
\hline rk & Param. & $\mu^{*}$ & $\mu$ & $\sigma$ \\
\hline 1 & rotation & 1.9702 & 1.9702 & $7.50 .10^{-3}$ \\
2 & centerX & 1.9050 & 1.9050 & $2.00 .10^{-3}$ \\
3 & radius & 0.9363 & -0.9363 & $2.65 .10^{-4}$ \\
4 & centerZ & 0.6460 & 0.6460 & $1.00 .10^{-6}$ \\
5 & $\mathrm{dx}$ & 0.2443 & -0.2443 & $1.08 .10^{-3}$ \\
6 & $\mathrm{dz}$ & 0.2248 & -0.2248 & $2.69 .10^{-4}$ \\
\hline
\end{tabular}

(c) Kurtosis at $70 \%$ MVC.

\begin{tabular}{|c|c|r|r|r|}
\hline rk & Param. & $\mu^{*}$ & $\mu$ & $\sigma$ \\
\hline 1 & rotation & 5.3278 & 5.3278 & $8.04 .10^{-2}$ \\
2 & centerX & 5.0865 & 5.0865 & $5.11 .10^{-2}$ \\
3 & radius & 2.5234 & -2.5234 & $1.21 .10^{-3}$ \\
4 & centerZ & 1.7485 & 1.7485 & $2.08 .10^{-4}$ \\
5 & $\mathrm{dx}$ & 0.6006 & -0.6006 & $6.33 .10^{-4}$ \\
6 & $\mathrm{dz}$ & 0.5714 & -0.5714 & $1.54 .10^{-3}$ \\
\hline
\end{tabular}

(b) Kurtosis at $50 \%$ MVC.

\begin{tabular}{|c|c|r|r|r|}
\hline rk & Param. & $\mu^{*}$ & $\mu$ & $\sigma$ \\
\hline 1 & rotation & 0.2886 & 0.2886 & $7.33 .10^{-4}$ \\
2 & centerX & 0.2459 & 0.2459 & $2.64 .10^{-6}$ \\
3 & radius & 0.1538 & -0.1538 & $1.61 .10^{-4}$ \\
4 & centerZ & 0.0410 & 0.0410 & $2.03 .10^{-8}$ \\
5 & $\mathrm{dx}$ & 0.0332 & -0.0332 & $1.15 .10^{-5}$ \\
6 & $\mathrm{dz}$ & 0.0118 & -0.0118 & $8.34 .10^{-6}$ \\
\hline
\end{tabular}

(d) Skewness at $30 \%$ MVC.

\begin{tabular}{|c|c|r|r|r|}
\hline rk & Param. & $\mu^{*}$ & $\mu$ & $\sigma$ \\
\hline 1 & centerZ & 0.0436 & -0.0436 & $9.60 .10^{-5}$ \\
2 & rotation & 0.0383 & 0.0383 & $1.00 .10^{-5}$ \\
3 & $\mathrm{dz}$ & 0.0303 & 0.0303 & $2.64 .10^{-4}$ \\
4 & centerX & 0.0276 & 0.0073 & $7.60 .10^{-4}$ \\
5 & $\mathrm{dx}$ & 0.0144 & 0.0144 & $2.60 .10^{-5}$ \\
6 & radius & 0.0129 & -0.0078 & $1.67 .10^{-4}$ \\
\hline
\end{tabular}

(e) Skewness at $50 \%$ MVC.

\begin{tabular}{|c|c|r|r|r|}
\hline rk & Param. & $\mu^{*}$ & $\mu$ & $\sigma$ \\
\hline 1 & centerZ & 0.0287 & -0.0287 & $4.80 .10^{-5}$ \\
2 & $\mathrm{dz}$ & 0.0152 & 0.0152 & $8.30 .10^{-5}$ \\
3 & rotation & 0.0151 & 0.0151 & $1.13 .10^{-4}$ \\
4 & centerX & 0.0116 & -0.0073 & $1.35 .10^{-4}$ \\
5 & radius & 0.0099 & -0.0026 & $9.80 .10^{-5}$ \\
6 & $\mathrm{dx}$ & 0.0051 & 0.0051 & $2.50 .10^{-5}$ \\
\hline
\end{tabular}

(f) Skewness at $70 \% \mathrm{MVC}$.

Table 6: Detailed bipolar kurtosis and skewness features sensitivity for all parameters on the mean features of the ten anatomies. Highlighted values in red correspond to values indicating a non monotonous effect of the parameter.

\subsection{Laplacian sensitivity ranking}

As we can see on the Fig 8 , like the monopolar and bipolar arrangements, the features are less sensitive to the lateral and longitudinal inter-electrode distance and the most sensitive to the rotation, the lateral center of the grid and the electrode radius. However, we observe different ranking of the parameters according to the anatomies. All the HOS features present different ranking for the two most sensitive parameters, the rotation and the lateral center of the grid. 


\begin{tabular}{|c|c|c|c|c|c|c|}
\hline \multirow[b]{2}{*}{ ARV_30 } & \multicolumn{6}{|c|}{ Parameter ranking according to sensitivity index (per feature) } \\
\hline & 2 & 4 & 5 & 6 & 3 & 1 \\
\hline ARV_50 & 2 & 4 & 5 & 6 & 3 & 1 \\
\hline ARV_70 & 2 & 4 & 5 & 6 & 3 & 1 \\
\hline RMS_30 & 2 & 6 & 4 & 5 & 3 & 1 \\
\hline RMS_50 & 2 & 5 & 4 & 6 & 3 & 1 \\
\hline RMS_70 & 2 & 5 & 4 & 6 & 3 & 1 \\
\hline Kurt_30 & 1 & 4 & 5 & 6 & 3 & 2 \\
\hline Kurt_50 & 1 & 4 & 6 & 5 & 3 & 2 \\
\hline Kurt_70 & 1 & 4 & 6 & 5 & 3 & 2 \\
\hline Skew_30 & 2 & 5 & 6 & 4 & 3 & 1 \\
\hline Skew_50 & 1 & 4 & 6 & 5 & 3 & 2 \\
\hline Skew_70 & 1 & 4 & 5 & 6 & 3 & 2 \\
\hline
\end{tabular}

Figure 8: Parameters ranking according to the features for laplacian arrangement on the grid.

On tables 7 and 8 , are presented the sensitivities values with their rank for all the parameters. We don't observe significant difference between the $\mu^{*}$ values, meaning that all the parameters have an impact on the given features. Also, no parameter has an important $\sigma$ value.

According to the values presented in tables 3 and 4 , we observe that the lateral center of the grid has higher impact on the HOS features (Kurtosis and Skewness) at 50 and $70 \% \mathrm{MVC}$ with the laplacian arrangement than with the monopolar and bipolar displays. However, according to the Table 8 the difference on the $\mu^{*}$ values between the parameters centerX and the rotation is minimal for all the HOS features for all contraction levels. Thus, the lateral center and the rotation of the grid are the two parameters with the highest impact on the HOS features for the laplacian configuration of the grid. 


\begin{tabular}{|c|c|r|r|r|}
\hline rk & Param. & $\mu^{*}$ & $\mu$ & $\sigma$ \\
\hline 1 & rotation & 0.1199 & 0.1199 & $3.67 .10^{-4}$ \\
2 & centerX & 0.1011 & 0.1011 & $4.21 .10^{-5}$ \\
3 & radius & 0.0663 & -0.0663 & $4.31 .10^{-5}$ \\
4 & centerZ & 0.0344 & 0.0344 & $2.84 .10^{-7}$ \\
5 & $\mathrm{dx}$ & 0.0259 & -0.0259 & $1.73 .10^{-7}$ \\
6 & $\mathrm{dz}$ & 0.0257 & -0.0257 & $1.17 .10^{-6}$ \\
\hline
\end{tabular}

(a) ARV at $30 \% \mathrm{MVC}$.

\begin{tabular}{|c|c|r|r|r|}
\hline rk & Param. & $\mu^{*}$ & $\mu$ & $\sigma$ \\
\hline 1 & rotation & 0.4999 & 0.4999 & $3.54 .10^{-3}$ \\
2 & centerX & 0.4416 & 0.4416 & $6.66 .10^{-4}$ \\
3 & radius & 0.2691 & -0.2691 & $2.85 .10^{-4}$ \\
4 & centerZ & 0.1481 & 0.1481 & $8.00 .10^{-6}$ \\
5 & $\mathrm{dx}$ & 0.1136 & -0.1136 & $1.40 .10^{-5}$ \\
6 & $\mathrm{dz}$ & 0.0862 & -0.0862 & $4.00 .10^{-6}$ \\
\hline
\end{tabular}

(c) ARV at $70 \% \mathrm{MVC}$.

\begin{tabular}{|c|c|r|r|r|}
\hline rk & Param. & $\mu^{*}$ & $\mu$ & $\sigma$ \\
\hline 1 & rotation & 0.2702 & 0.2702 & $3.70 .10^{-3}$ \\
2 & centerX & 0.2242 & 0.2242 & $7.25 .10^{-4}$ \\
3 & radius & 0.1430 & -0.1430 & $2.56 .10^{-4}$ \\
4 & $\mathrm{dx}$ & 0.0851 & -0.0851 & $4.53 .10^{-9}$ \\
5 & centerZ & 0.0802 & 0.0802 & $1.15 .10^{-5}$ \\
6 & $\mathrm{dz}$ & 0.0588 & -0.0588 & $4.17 .10^{-7}$ \\
\hline
\end{tabular}

(e) RMS at $50 \%$ MVC.

\begin{tabular}{|c|c|r|r|r|}
\hline rk & Param. & $\mu^{*}$ & $\mu$ & $\sigma$ \\
\hline 1 & rotation & 0.3389 & 0.3389 & $2.00 .10^{-3}$ \\
2 & centerX & 0.2969 & 0.2969 & $3.87 .10^{-4}$ \\
3 & radius & 0.1843 & -0.1843 & $1.59 .10^{-4}$ \\
4 & centerZ & 0.0999 & 0.0999 & $4.00 .10^{-6}$ \\
5 & $\mathrm{dx}$ & 0.0822 & -0.0822 & $7.00 .10^{-6}$ \\
6 & $\mathrm{dz}$ & 0.0582 & -0.0582 & $3.00 .10^{-6}$ \\
\hline
\end{tabular}

(b) ARV at $50 \% \mathrm{MVC}$.

\begin{tabular}{|c|c|r|r|r|}
\hline rk & Param. & $\mu^{*}$ & $\mu$ & $\sigma$ \\
\hline 1 & rotation & 0.0249 & 0.0249 & $5.99 .10^{-5}$ \\
2 & centerX & 0.0185 & 0.0185 & $6.04 .10^{-6}$ \\
3 & radius & 0.0141 & -0.0141 & $7.09 .10^{-6}$ \\
4 & $\mathrm{dx}$ & 0.0074 & -0.0074 & $3.43 .10^{-7}$ \\
5 & $\mathrm{dz}$ & 0.0072 & -0.0072 & $1.82 .10^{-10}$ \\
6 & centerZ & 0.0067 & 0.0067 & $1.17 .10^{-8}$ \\
\hline
\end{tabular}

(d) RMS at $30 \%$ MVC.

\begin{tabular}{|c|c|r|r|r|}
\hline rk & Param. & $\mu^{*}$ & $\mu$ & $\sigma$ \\
\hline 1 & rotation & 0.4587 & 0.4587 & $9.89 .10^{-3}$ \\
2 & centerX & 0.3810 & 0.3810 & $1.78 .10^{-3}$ \\
3 & radius & 0.2409 & -0.2409 & $7.48 .10^{-4}$ \\
4 & $\mathrm{dx}$ & 0.1385 & -0.1385 & $4.16 .10^{-6}$ \\
5 & centerZ & 0.1355 & 0.1355 & $2.66 .10^{-5}$ \\
6 & $\mathrm{dz}$ & 0.1008 & -0.1008 & $2.38 .10^{-7}$ \\
\hline
\end{tabular}

(f) RMS at $70 \% \mathrm{MVC}$.

Table 7: Detailed laplacian ARV and RMS features sensitivity for all parameters on the mean features of the ten anatomies. Highlighted values in red correspond to values indicating a non monotonous effect of the parameter. 


\begin{tabular}{|c|c|r|r|r|}
\hline rk & Param. & $\mu^{*}$ & $\mu$ & $\sigma$ \\
\hline 1 & centerX & 2.6132 & 2.6132 & $5.27 .10^{-3}$ \\
2 & rotation & 2.6039 & 2.6039 & $2.57 .10^{-4}$ \\
3 & radius & 1.4080 & -1.4080 & $4.27 .10^{-3}$ \\
4 & centerZ & 0.7421 & 0.7421 & $5.10 .10^{-3}$ \\
5 & $\mathrm{dx}$ & 0.3921 & -0.3921 & $3.54 .10^{-3}$ \\
6 & $\mathrm{dz}$ & 0.1984 & -0.1984 & $6.08 .10^{-4}$ \\
\hline
\end{tabular}

(a) Kurtosis at $30 \%$ MVC.

\begin{tabular}{|c|c|r|r|r|}
\hline rk & Param. & $\mu^{*}$ & $\mu$ & $\sigma$ \\
\hline 1 & centerX & 4.2755 & 4.2755 & $1.62 .10^{-2}$ \\
2 & rotation & 4.1861 & 4.1861 & $1.99 .10^{-1}$ \\
3 & radius & 1.9711 & -1.9711 & $2.21 .10^{-2}$ \\
4 & centerZ & 1.3503 & 1.3503 & $4.74 .10^{-4}$ \\
5 & $\mathrm{dz}$ & 0.4327 & -0.4327 & $8.31 .10^{-4}$ \\
6 & $\mathrm{dx}$ & 0.2104 & -0.2104 & $7.17 .10^{-4}$ \\
\hline
\end{tabular}

(c) Kurtosis at $70 \%$ MVC.

\begin{tabular}{|c|c|r|r|r|}
\hline rk & Param. & $\mu^{*}$ & $\mu$ & $\sigma$ \\
\hline 1 & centerX & 1.5228 & 1.5228 & $1.65 .10^{-7}$ \\
2 & rotation & 1.5109 & 1.5109 & $7.28 .10^{-3}$ \\
3 & radius & 0.7995 & -0.7995 & $2.32 .10^{-3}$ \\
4 & centerZ & 0.4971 & 0.4971 & $1.67 .10^{-5}$ \\
5 & $\mathrm{dz}$ & 0.1617 & -0.1617 & $1.71 .10^{-3}$ \\
6 & $\mathrm{dx}$ & 0.1022 & -0.1022 & $3.71 .10^{-3}$ \\
\hline
\end{tabular}

(e) Skewness at $50 \% \mathrm{MVC}$.

\begin{tabular}{|c|c|r|r|r|}
\hline rk & Param. & $\mu^{*}$ & $\mu$ & $\sigma$ \\
\hline 1 & centerX & 9.6200 & 9.6200 & $2.32 .10^{-2}$ \\
2 & rotation & 9.6013 & 9.6013 & $3.79 .10^{-1}$ \\
3 & radius & 4.7833 & -4.7833 & $7.59 .10^{-2}$ \\
4 & centerZ & 3.0745 & 3.0745 & $1.00 .10^{-6}$ \\
5 & $\mathrm{dz}$ & 1.0773 & -1.0773 & $1.39 .10^{-2}$ \\
6 & $\mathrm{dx}$ & 0.5127 & -0.5127 & $1.72 .10^{-2}$ \\
\hline
\end{tabular}

(b) Kurtosis at $50 \% \mathrm{MVC}$.

\begin{tabular}{|c|c|r|r|r|}
\hline rk & Param. & $\mu^{*}$ & $\mu$ & $\sigma$ \\
\hline 1 & rotation & 0.1995 & 0.1995 & $1.98 .10^{-3}$ \\
2 & centerX & 0.1884 & 0.1884 & $1.29 .10^{-3}$ \\
3 & radius & 0.1184 & -0.1184 & $6.00 .10^{-6}$ \\
4 & $\mathrm{dz}$ & 0.0498 & -0.0498 & $1.20 .10^{-5}$ \\
5 & centerZ & 0.0306 & 0.0306 & $8.00 .10^{-5}$ \\
6 & $\mathrm{dx}$ & 0.0175 & 0.0059 & $3.05 .10^{-4}$ \\
\hline
\end{tabular}

(d) Skewness at $30 \% \mathrm{MVC}$.

\begin{tabular}{|c|c|r|r|r|}
\hline rk & Param. & $\mu^{*}$ & $\mu$ & $\sigma$ \\
\hline 1 & centerX & 1.1506 & 1.1506 & $6.49 .10^{-4}$ \\
2 & rotation & 1.1398 & 1.1398 & $9.24 .10^{-3}$ \\
3 & radius & 0.5641 & -0.5641 & $1.27 .10^{-3}$ \\
4 & centerZ & 0.3626 & 0.3626 & $1.52 .10^{-4}$ \\
5 & $\mathrm{dx}$ & 0.1055 & -0.1055 & $3.94 .10^{-4}$ \\
6 & $\mathrm{dz}$ & 0.0999 & -0.0999 & $4.04 .10^{-4}$ \\
\hline
\end{tabular}

(f) Skewness at $70 \% \mathrm{MVC}$.

Table 8: Detailed laplacian kurtosis and skewness features sensitivity for all parameters on the mean features of the ten anatomies. Highlighted values in red correspond to values indicating a non monotonous effect of the parameter.

According to all these results, the specific parameters about the layout of the HD grid (inter-electrode distances) have a minor effect compared to parameters specific to the HD grid position (rotation and center of the grid) for monopolar, bipolar and laplacian arrangements. Therefore, HD-sEMG applications should have a specific focus on the right alignment with the muscle fibers and on the good positioning of the lateral center of the grid. Finally, we saw that the parameter sensitiveness is also depending on the anatomy. According to the obtained results, HOS features seem to be able to provide some information about the nature of the activated muscle region. 


\section{Discussion \& Conclusion}

The objective of this study was to investigate and to precisely quantify the sensitivity of HD-sEMG amplitude descriptors according to varying parameters related to the HD-sEMG grid design and position. This interest is motivated by recent studies using HD-sEMG grid [19, 20]. For this purpose, we proposed a first global sensitivity analysis based on the EEM. The 64 simulated signals per grid are generated using a recent multilayered cylinder muscle model [1]. This model allowed us to compute all the signals on the same potential surface map provided by the DOE definition which describes the different electrode grid to model, from the EEM. This modelization formalism allows us to study only the electrode grid effect on the generated signals. In total, 16 electrode grid composed of 64 electrodes are defined and computed for each contraction level and anatomy. This gave us $5 \times 16 \times 64$ signals for this study. Also, monopolar, bipolar and laplacian arrangements on the grid are tested for this analysis. Moreover, 10 different anatomies were generated with the same properties (number of MUs, adipose and skin tissue conductivities and thicknesses) but with different position of the MUs within the muscle. We did this in order to do a robust analysis of the parameters sensitivity and to investigate the possible influence of the MU positions on the sensitivity measures. The main result of this study is the identification of the tested parameter that has the higher impact on the amplitude descriptors: the grid rotation. A misalignement of the grid with muscle fibers will have much more effect on the recorded HD-sEMG signals than having an increase of the lateral inter-electrode distance. Other parameters with high impact are the lateral grid position and the electrode radius variation. Thus, we assessed that the HD-sEMG grid layout (inter-electrode distances) induces lower significant deviations on the signals than the grid position. Furthermore, placement of MUs within the muscle has a little effect on the classical parameters sensitivity. However, HOS features, especially at lower contraction levels, seem to be sensitive to the MU placement particularly with monopolar and laplacian configurations. According to the results, we also assessed that the ARV and RMS features in monopolar arrangement have the same sensitivity and thus are equivalent for our study. Finally, future works will focus on the testing of frequency features in complement of the amplitude descriptors of the HD-sEMG signals. Actually, those features have also desmonstrated their usefulness in assessing muscle fatigue and neuromuscular diseases [21].

This study has been performed with an analytical model using a multilayered cylinder muscle with a high spatial resolution of the surface maps and a high number of simulated MUs. This kind of study is useful for helping practitioners in designing efficient instrumental protocols that take into account the results of such sensitivity analysis. In fact, they have to be careful about the grid placement according to the muscle fibers orientation. A misalignment of a few degree will bias the recording signals between the subjects and thus, give false interpretation of the results. This result is in agreement with a previous study [22] where authors only studied the rotation effect of 
the grid according to the fiber orientation. Also, the lateral center of the grid has to be carefully chosen. On the same subject, an other adjustement of the lateral grid center between two recordings will induce a different investigation region of the muscle. Thus, different MUs will be examined and different results will be obtained. Future sensitivity analysis studies, using the described HD-sEMG model, will focus on both anatomical and neural parameters of the muscle to evaluate their effects on the amplitude descriptors.

\section{Acknowledgements}

This work was carried out in the framework of the Labex MS2T, which was funded by the French Government, through the program "Investments for the future" managed by the National Agency for Research (Reference ANR-11-IDEX-0004-02).

\section{Conflict of interest}

The authors declare that they have no conflict of interest.

\section{References}

[1] V. Carriou, S. Boudaoud, J. Laforet, and F. S. Ayachi, "Fast generation model of high density surface EMG signals in a cylindrical conductor volume," Computers in Biology and Medicine, vol. 74, pp. 54-68, July 2016.

[2] T. Heidlauf and O. Rohrle, "Modeling the Chemoelectromechanical Behavior of Skeletal Muscle Using the Parallel Open-Source Software Library OpenCMISS," Computational and Mathematical Methods in Medicine, vol. 2013, Nov. 2013.

[3] R. Merletti and P. A. Parker, Electromyography: Physiology, Engineering, and Non-Invasive Applications. John Wiley \& Sons, July 2004.

[4] E. A. Clancy and N. Hogan, "Probability density of the surface electromyogram and its relation to amplitude detectors," IEEE transactions on bio-medical engineering, vol. 46, pp. 730-739, June 1999.

[5] F. Ayachi, S. Boudaoud, J. F. Grosset, and C.Marque, "Study of the Muscular Force/HOS Parameters Relationship from the Surface Electromyogram," in 15th Nordic-Baltic Conference on Biomedical Engineering and Medical Physics, vol. 34, pp. 187-190, Springer Berlin Heidelberg, 2011.

[6] M. D. Morris, "Factorial Sampling Plans for Preliminary Computational Experiments," Technometrics, vol. 33, no. 2, pp. 161-174, 1991.

[7] V. Carriou, M. Al Harrach, J. Laforet, and S. Boudaoud, "Sensitivity Analysis of HDsEMG Amplitude Descriptors Relative to Grid Parameter Variation," in XIV Mediterranean Conference on Medical and Biological Engineering and Computing, vol. 57, pp. 119-123, Springer International Publishing, 2016.

[8] J. Laforet and C. Marque, "Preliminary global sensitivity analysis of a uterine electrical activity model," in 35th Annual International Conference of the IEEE Engineering in Medicine and Biology Society, 2013.

[9] S. Schiaffino and C. Reggiani, "Fiber Types in Mammalian Skeletal Muscles," Physiological Reviews, vol. 91, no. 4, pp. 1447-1531, 2011.

[10] V. Carriou, J. Laforet, S. Boudaoud, and M. Al Harrach, "Realistic motor unit placement in a cylindrical HD-sEMG generation model," in 38th Annual International Conference of the IEEE Engineering in Medicine and Biology Society. Orlando, USA., 2016 (accepted). 
[11] C. S. Klein, G. D. Marsh, R. J. Petrella, and C. L. Rice, "Muscle fiber number in the biceps brachii muscle of young and old men," Muscle \&3 Nerve, vol. 28, no. 1, pp. 62-68, 2003.

[12] T. I. Arabadzhiev, V. G. Dimitrov, N. A. Dimitrova, and G. V. Dimitrov, "Influence of motor unit synchronization on amplitude characteristics of surface and intramuscularly recorded EMG signals," European Journal of Applied Physiology, vol. 108, no. 2, pp. 227-237, 2009.

[13] C. J. D. Luca and P. Contessa, "Hierarchical control of motor units in voluntary contractions," Journal of Neurophysiology, vol. 107, no. 1, pp. 178-195, 2012.

[14] F. S. Ayachi, S. Boudaoud, and C. Marque, "Evaluation of muscle force classification using shape analysis of the sEMG probability density function: a simulation study," Medical \&3 Biological Engineering \& Computing, vol. 52, no. 8, pp. 673-684, 2014.

[15] A. Saltelli and F. Campolongo, "Screening important inputs in models with strong interaction properties," Reliability Engineering EGamp; System Safety, vol. 94, no. 7, pp. 1149-1155, 2009.

[16] A. Saltelli, Global sensitivity analysis: the primer. John Wiley, 2008.

[17] M. Al Harrach, S. Boudaoud, M. Hassan, F. S. Ayachi, D. Gamet, J. F. Grosset, and F. Marin, "Denoising of HD-sEMG signals using canonical correlation analysis," Medical \&s Biological Engineering \& Computing, pp. 1-14, 2016.

[18] M. Al Harrach, S. Boudaoud, D. Gamet, J. F. Grosset, and F. Marin, "Evaluation of HD-sEMG Probability Density Function deformations in ramp exercise," in 36th Annual International Conference of the IEEE Engineering in Medicine and Biology Society, pp. 2209-2212, IEEE, 2014.

[19] S. Boudaoud, S. Allouch, M. Al Harrach, and F. Marin, "On the benefits of using HD-sEMG technique for estimating muscle force," Computer Methods in Biomechanics and Biomedical Engineering, vol. 18, pp. 1890-1891, 2015.

[20] E. M. Maathuis, J. Drenthen, J. P. van Dijk, G. H. Visser, and J. H. Blok, "Motor unit tracking with high-density surface EMG," Journal of Electromyography and Kinesiology, vol. 18, pp. 920930, 2008.

[21] A. Phinyomark, S. Thongpanja, H. Hu, P. Phukpattaranont, and C. Limsakul, "The Usefulness of Mean and Median Frequencies in Electromyography Analysis," in Computational Intelligence in Electromyography Analysis - A Perspective on Current Applications and Future Challenges (G. R. Naik, ed.), InTech, 2012.

[22] A. Zaylaa, A. Diad, M. Al Harrach, and S. Boudaoud, "Evaluation of HD-sEMG grid misalignment with muscle fibers using nonlinear correlation," in IEEE International Conference on Advances in Biomedical Engineering, pp. 289-292, 2015. 